\section{Detecting risk and predicting patient mortality in patients with extended-spectrum $\beta$-lactamase- producing Enterobacteriaceae bloodstream infections}

\author{
Enrico Maria Trecarichi', Roberto Cauda' \& Mario Tumbarello*1 \\ Institute of Infectious Diseases, Catholic University of the Sacred Heart, A Gemelli Hospital, Rome, Italy \\ *Author for correspondence: Tel..: +39 0630155373 m. Fax: +39 063054519 m. tumbarello@rm.unicatt. it
}

Extended-spectrum $\beta$-lactamases (ESBLs) have been increasingly described worldwide, especially among Enterobacteriaceae isolates, and recently not only in the nosocomial, but also in the community setting. Bloodstream infections (BSIs) caused by ESBL-producing Enterobacteriaceae have been associated with increased rates of treatment failure, mortality and hospital costs. Any delay in the initiation of adequate antibiotic therapy is potentially lethal for patients with BSIs caused by ESBL-producing Enterobacteriaceae. The awareness of changes in bacterial resistance patterns, the careful knowledge of risk factors for ESBL infection and of factors facilitating adverse outcome, giving attention to local epidemiology, can improve the efficacy of empirical treatment protocols. The aim of this review is to focus on the main characteristics of BSIs caused by ESBLproducing Enterobacteriaceae, with particular emphasis on risk factors for these infections and factors related to mortality.

Extended-spectrum $\beta$-lactamases (ESBLs) are plasmid-mediated $\beta$-lactamases that confer resistance to oxyimino-cephalosporins and monobactams and have been increasingly described worldwide since their first description in the early 1980s, especially among Enterobacteriaceae isolates, and recently not only in the nosocomial, but also in the community setting [1-3].

The rate of ESBL production among Enterobacteriaceae varies worldwide. According to data reported by the European Antimicrobial Resistance Surveillance Network [101], the resistance rate to third-generation cephalosporins (mostly related to ESBL production) of Klebsiella pneumoniae (Kp) and Escherichia coli (Ec) clinical isolates collected in 28 European countries were 27.5 and $8.5 \%$, respectively, with proportions of resistant isolates in the reporting countries ranging from 2.6 to $24.8 \%$ for Ec and from 1.7 to $75.6 \%$ for $\mathrm{Kp}$.

Bloodstream infections (BSIs) caused by ESBLproducing Enterobacteriaceae have been associated with increased rates of treatment failure, mortality and hospital costs $[1,4,5]$. The presence of an ESBL determinant significantly reduces the number of antibiotic drugs to which the infecting organism is susceptible and, particularly in case of nosocomial infections, ESBL genes are often associated with other resistance genes, thus generating complex multidrug-resistant phenotypes of isolates and frequently causing a delay in initiation of adequate antimicrobial therapy $[2,6,7]$.

The aim of this review is to focus on the main characteristics of BSIs caused by ESBLproducing Enterobacteriaceae, with particular emphasis on risk factors for these infections and factors related to mortality.

A literature search was performed using the PubMed database through to 25 April 2012, in order to identify studies investigating risk factors for ESBL-producing Enterobacteriaceae BSIs and factors associated with mortality for these infections. Searches were performed using the following search terms: '(bacteremia OR bloodstream) AND (ESBL OR extended-spectrum beta-lactamase OR extended-spectrum $\beta$-lactamase). Only papers published in English were considered.

\section{Risk factors for BSIs caused by ESBL-producing Enterobacteriaceae}

Several studies have been conducted to identify risk factors for BSIs caused by ESBL-producing Enterobacteriaceae; only 30 studies with multivariable regression models have been included, and principal data reported in these studies are shown in Table 1 [8-37].

In general, there is a large heterogeneity among these studies. Firstly, they include different

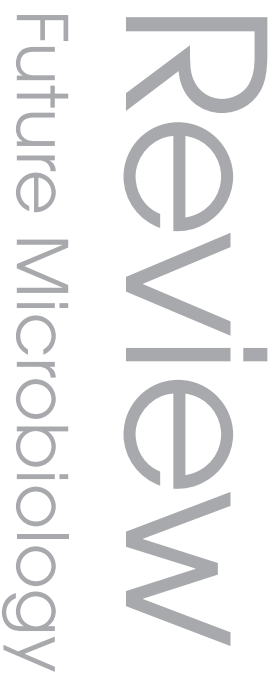

\section{Keywords \\ - bloodstream infection - Enterobacteriaceae = ESBL - Escherichia coli $=$ extended- spectrum $\beta$-lactamase - Klebsiella pneumonia - prognostic indicator = risk factor}

\section{Future : Medicine part of}




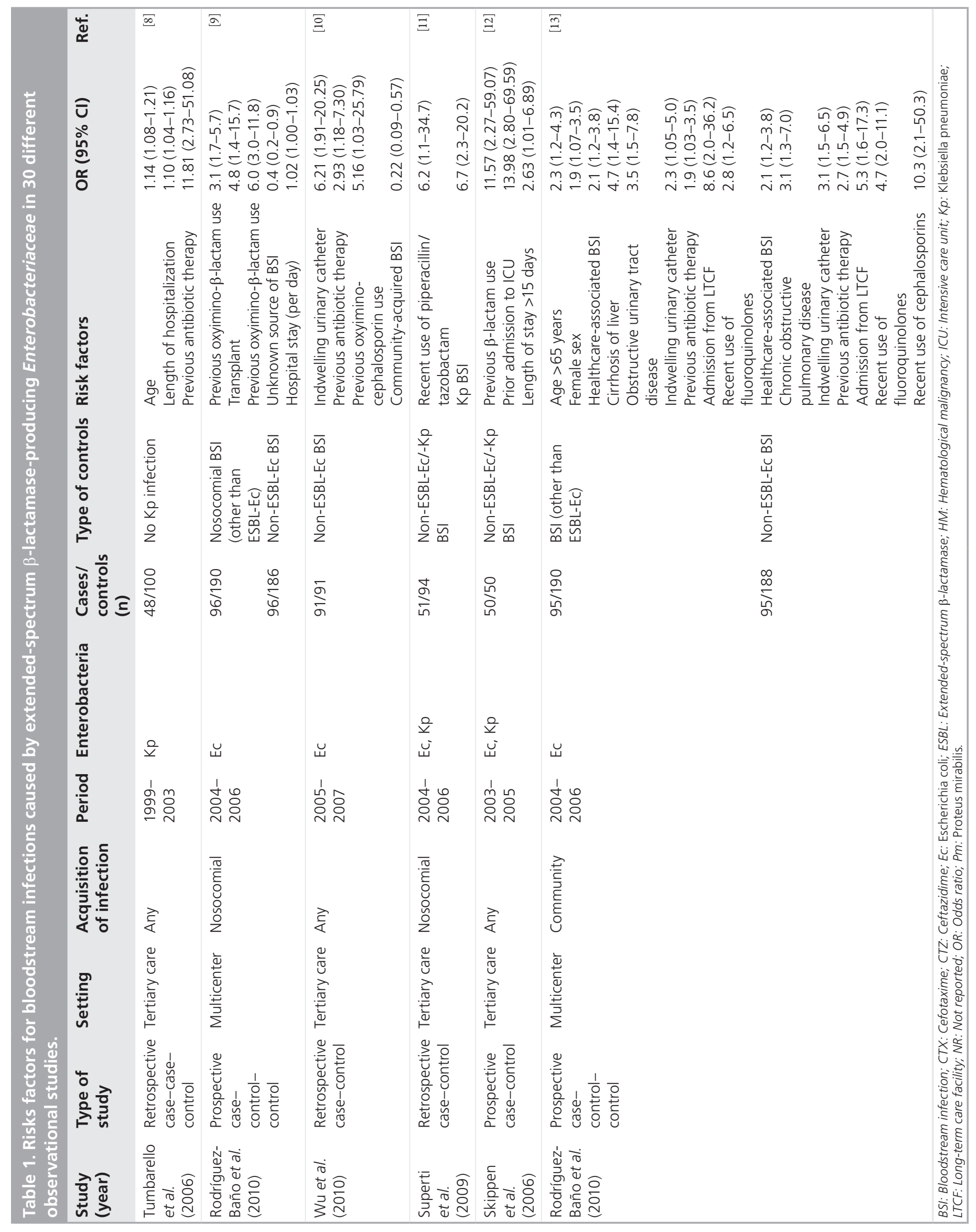




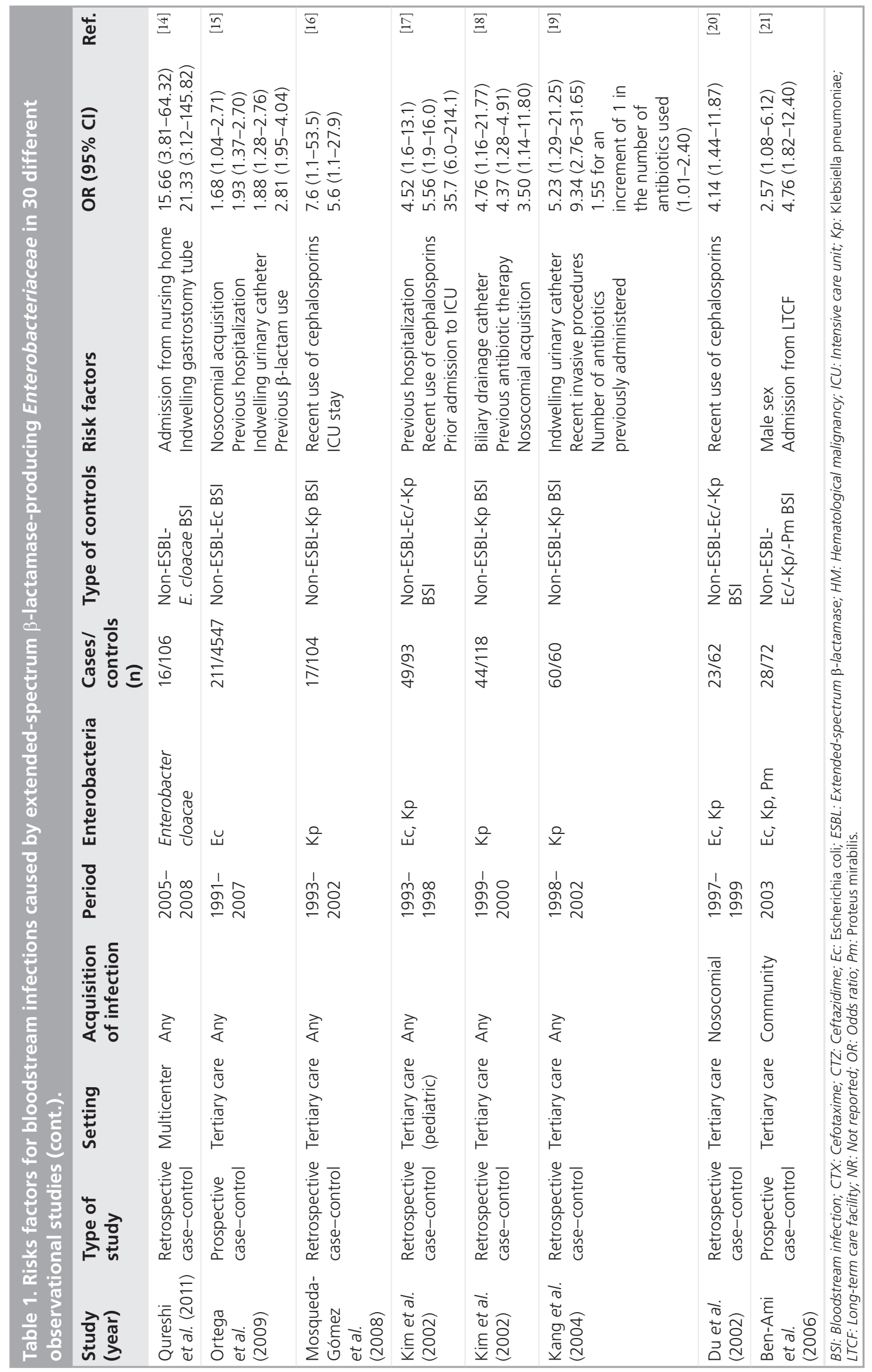




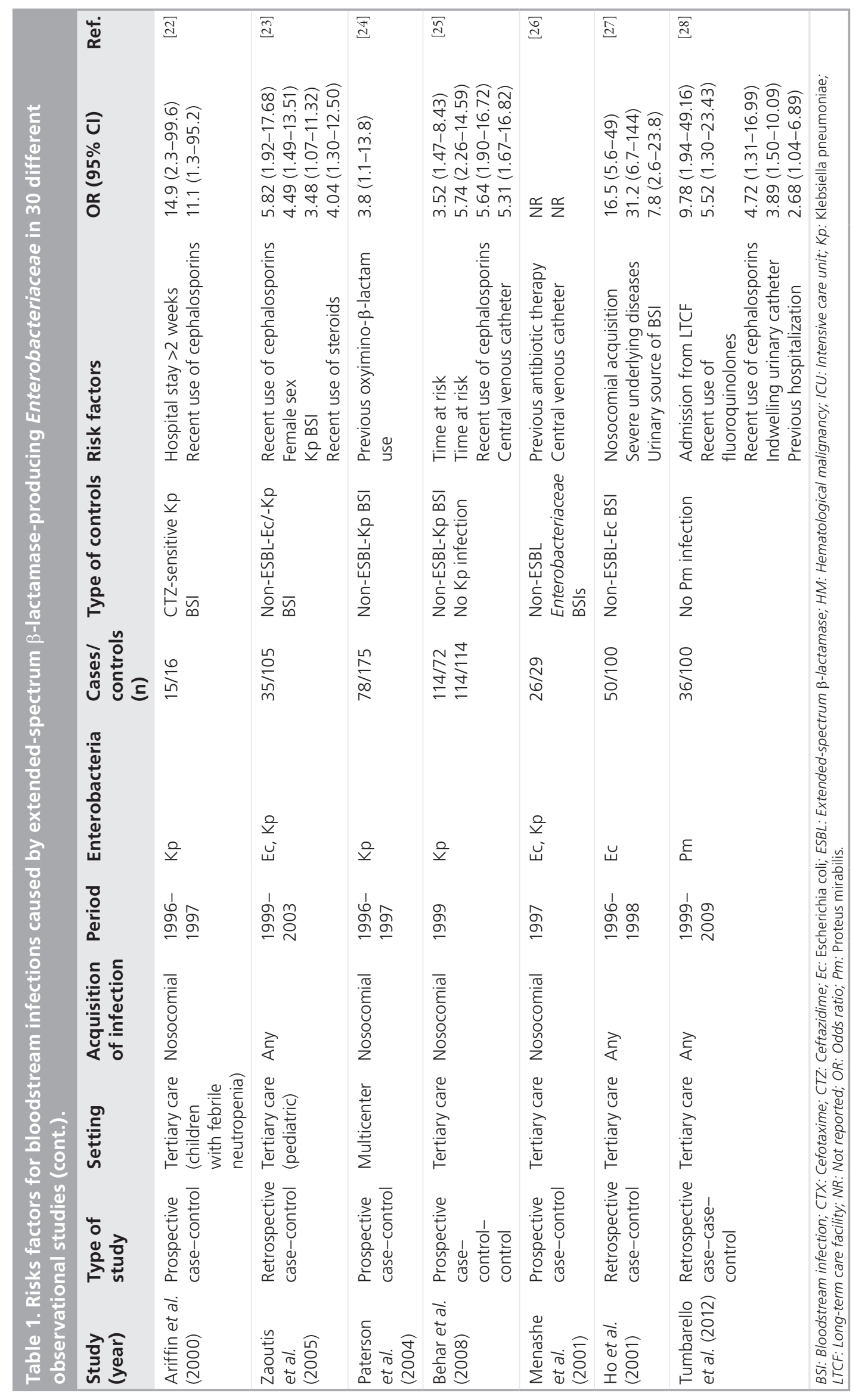




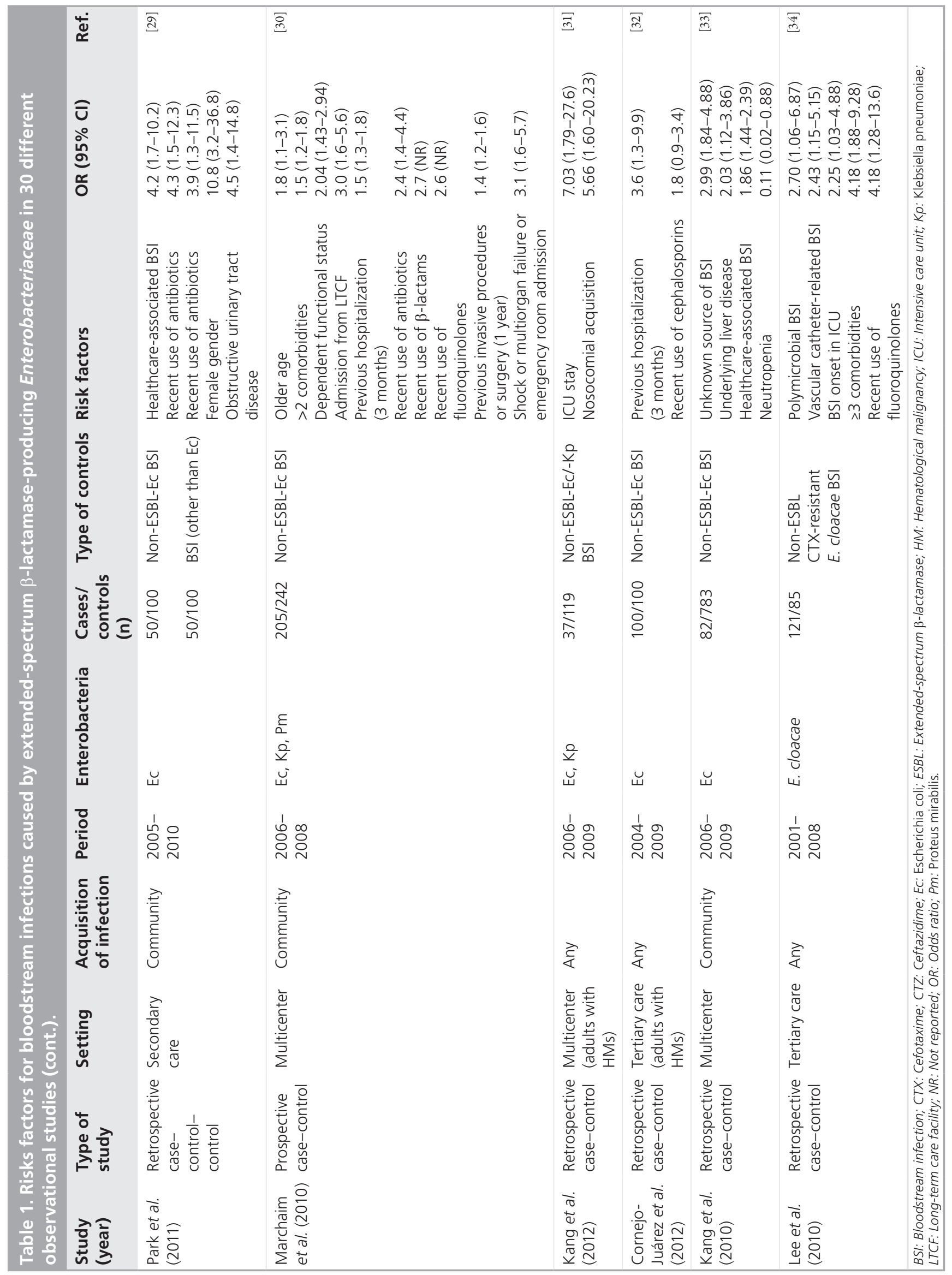




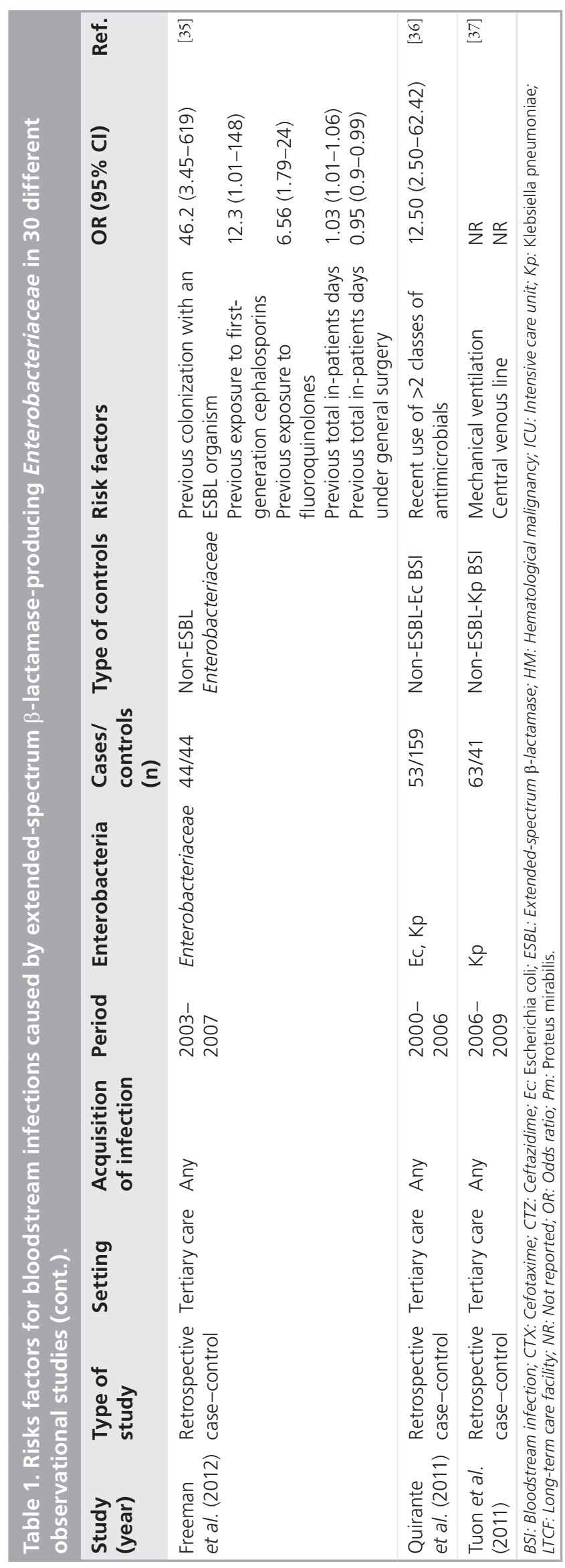

species of Enterobacteriaceae: eight studies have been conducted on Kp BSIs [8,16,18,19,22,24,25,37], eight on Ec BSIs [9,10,13,15,27,29,32,33], one on Proteus mirabilis (Pm) BSIs [28], seven on Ec and Kp BSIs $[11,12,17,20,23,31,36]$, two on Ec, Kp and Pm BSIs [21,30], two on all species of Enterobacteriaceae [26,35] and two on Enterobacter cloacae BSIs [14,34]. Secondly, acquisition of BSI (i.e., communityacquired, healthcare-associated and nosocomial) was different among the studies: 18 studies included cases with any type of BSI acquisition $[8,10,12,14-19,23,27,28,31,32,34-37]$, whereas seven included only nosocomial BSIs [9,11,20,22,24-26] and five only community-acquired BSIs $[13,21,29,30,33]$. Thirdly, only ten $[9,12,13,15,21,22,24-26,30]$ out of the 30 studies included have been conducted prospectively. These differences could be important because of the different epidemiology of the populations studied or the accuracy of data included. In this regard, the spread in the past decade of ESBL-producing Ec strains encoding CTX-M enzymes has been reported, particularly in nonhospitalized patients [13,21], with frequencies of ESBL enzymes belonging to this family of up to $87 \%$ in a cohort of patients with community-acquired ESBL-producing Ec BSIs [13], thus suggesting that the epidemiology of Enterobacteriaceae isolates encoding CTX-M enzymes could be different from those encoding the other ESBLs. Recently, Wu and colleagues have analyzed patients with Ec BSIs caused by strains that produce CTX-M or non-CTX-M ESBLs and found that chronic renal failure and intensive care unit (ICU) stay were independent predictors of CTX-M ESBL-producing Ec BSIs [38].

Finally, patients with BSIs caused by nonESBL-producing Enterobacteriaceae have been used as control groups in all except two studies, both by Tumbarello et al., in which patients with no evidence of Enterobacteriaceae (i.e., Kp and $\mathrm{Pm}$, respectively) infection were used in a double case-control study design $[8,28]$, whereas in the other four studies with a case-double control design, patients with BSIs caused by bacteria other than Enterobacteriaceae [30] or other than ESBL-producing Enterobacteriaceae $[9,13]$ and patients without cultures positive for Enterobacteriaceae [25] were also used as control groups. The importance of the selection of an appropriate control group for studies investigating risk factors for antibiotic-resistant bacteria infections has been previously outlined, and differences in types and magnitude of identified risk factors could depend on the different control groups used. In this regard, Harris 
et al. highlighted that in studies investigating risk factors for infections caused by antibioticresistant bacteria, a control group consisting of patients with the susceptible form of the same organism (as in most of the studies included in the present review) might lead to an overestimation of the importance of previous antimicrobial use [39]. More generally, a distinction could be made between a prognostic approach (when the question to be answered is: 'Which patients with suspected BSIs are infected with an ESBL-producing organism?') and an etiologic approach (when the questions to be answered are: 'What drives the spread of ESBLs in clinical patients?' or 'What are the risk factors for acquiring ESBL-producing bacteria in different settings [e.g., hospital or community]?'); the source population for the first question would be patients with a BSI caused by the susceptible form of the same organism or by a different pathogen, and for the latter questions, it would be patients belonging to the same setting (i.e., hospitalized or community patients) [25,39].

Given the above, seven studies identified recent antibiotic therapy as a risk factor for ESBL-producing Enterobacteriaceae BSIs, with odds ratios (ORs) ranging from 1.9 to 11.81 $[8,10,13,18,26,29,30]$. In a study by Kang et al., the number of antibiotics previously administered was independently associated with BSIs caused by ESBL-producing Kp, with an OR of 1.55 for an increment of 1 in the number of antibiotics used [19]. Similarly, in a study by Quirante et al., recent use of more than two classes of antimicrobials was found to increase the risk of ESBL-producing Ec or Kp BSIs by approximately 12 times [36]. In the case double-control study of Park et al., comparison with both control groups revealed that the recent use of antibiotics was the only independent risk factor for ESBL acquisition [29].

When single antibiotic classes were analyzed, recent use of $\beta$-lactams was identified as a risk factor for ESBL-producing Enterobacteriaceae BSIs, with ORs ranging from 1.8 to 12.3 , in 17 studies [9-13,15-17,20,22-25,28,30,32,35]; in particular, in 11 of these studies, the oxyimino-cephalosporins were the previous $\beta$-lactams used $[10,13,16,17,20,22,23,25,28,32,35]$. In a survey of $13 \mathrm{dif}-$ ferent hospitals conducted by Rodríguez-Baño et al., previous fluoroquinolones use was associated with community-acquired ESBL-producing Ec BSIs in two case-control studies involving as controls patients with community-acquired BSIs caused by bacteria other than ESBLproducing Ec and by non-ESBL-producing Ec, respectively [13]. Previous use of fluoroquinolones has also been reported as an independent risk factor for ESBL-producing Enterobacteriaceae BSIs in four other studies $[28,30,34,35]$.

Factors related to previous hospitalization have been found to be independently associated with ESBL-producing Enterobacteriaceae BSIs in several studies [8-10,12-18,21,22,25,28-35]. In particular, a prolonged length of hospital stay has been reported to increase the risk of ESBL-producing Enterobacteriaceae compared with controls, with ORs ranging from 1.03 to 14.9 [8,12,15,17,22,25,35]; an increment of OR for ESBL-producing Ec BSIs of 1.02 per day of hospital stay was found in a study by Rodríguez-Baño et al. [9], and previous stay in an ICU was found to increase the risk up to 35.7 times $[12,16,17]$. In other studies, nosocomial acquisition and healthcare-associated acquisition of BSIs have been recognized as more likely to occur in ESBL-producing Enterobacteriaceae BSIs $[13,15,18,27,29,31,33]$, and, conversely, community-acquired infection has been reported to be a negative predictor for ESBL production [10].

The third important group of risk factors that have been associated with ESBL-producing Enterobacteriaceae BSIs includes previous invasive procedures and/or presence of devices $[10,13-15,18,19,25,26,28,30,34,37]$. In particular, urinary catheterization has been found to be an independent risk factor in four studies, increasing the risk of BSIs caused by ESBL-producing Enterobacteriaceae by $1.88-6.21$ times $[10,13,15,19]$; other devices identified in individual studies were gastrostomy tubes [14], biliary drainage catheters [18], mechanical ventilators [37] and central venous catheters $[25,26,34,37]$.

Factors related to the patient's clinical status and underlying diseases, such as old age, transplant, liver cirrhosis and chronic obstructive pulmonary disease, have also been reported to increase the likelihood of ESBL-producing Enterobacteriaceae BSIs [8,9,13,27,29,30,33,34].

In recent years, ESBL-producing Enterobacteriaceae have been increasingly reported to be pathogens in community-acquired infections $[1,3,13,21,29,30,33,40]$, and this has important implications for the management of these infections, particularly concerning the choice of efficacious empirical treatment for those that are serious. Risk factors for non-nosocomial ESBL-producing Enterobacteriaceae BSIs have been specifically investigated in five studies $[13,21,29,30,33]$. In the case-control-control study of Rodríguez-Baño et al., a number of risk factors were identified, which were slightly different depending on the 
control group used and included the patients' recent use of antibiotics or not (i.e., fluoroquinolones and/or cephalosporins), presence of urinary catheter and presence of comorbidities (i.e., chronic obstructive pulmonary disease and/ or liver chirrosis) [13]. Interestingly, in this study, for both of the control groups used and in those of Ben-Ami et al. [21] and Marchaim et al. [30], admission from long-term care facilities (LTCFs) was among the strongest risk factors. In the study of Qureshi et al. involving 122 cases of E. cloacae BSI of which 46 (37.7\%) were non-nosocomial, admission from a nursing home remained the most important risk factor for ESBL-producing isolate infection (OR: 15.66) [14]. Similarly, admission from LTCFs was found to increase the likelihood of ESBL-producing Pm BSIs by approximately ten times in a study by Tumbarello et al., including 99 ESBL-producing Pm BSIs, of which 17 (seven ESBL-producing Pm BSIs and ten non-ESBLproducing Pm BSIs) were detected $<48 \mathrm{~h}$ postadmission [28]. LTCFs have frequently been singled out as important reservoirs of ESBL-producing Enterobacteriaceae in different countries [2,41], and cases of serious infections acquired outside hospitals that might be caused by Enterobacteriaceae (e.g., complicated urinary tract infections and intra-abdominal infections) in patients admitted from LTCFs should be highly suspected as being caused by ESBL-producing isolates. In addition, Tumbarello et al. have recently published a scoring system for identifying patients harboring ESBL-producing Enterobacteriaceae at hospital admission; in the case group, $21.4 \%$ of patients had a BSI, and admission from another healthcare facility (including LTCFs) had the highest score point [40]. The complex dynamics behind ESBLproducing Enterobacteriaceae colonization in the community must also be highlighted, which involves reservoirs not only related to contact with healthcare facilities, but also with foreign travels, contact with animals and meat consumption, all of which being aided by antibiotic use in individuals [1-3].

\section{Predictors for mortality in patients with BSIs caused by ESBL-producing Enterobacteriaceae}

Only cohort studies in which multivariable regression models have been conducted in order to identify risk factors for mortality in patients suffering from BSIs caused by Enterobacteriaceae have been included in this review. However, the outcome of ESBL-producing Enterobacteriaceae BSIs might generally be the result of a complex interaction of multiple determinants, which might be considered both confounders and/or intermediates, and adjustment in both cases might affect the true causal relationship, even in multivariable models [42].

The mortality rate for patients with BSIs caused by Enterobacteriaceae ranged from 8.1 to $43.6 \%$ in the 28 studies included. The principal data found in these studies are reported in Table 2 [9,13,15,18-20,26,28-31,33,34,43-57].

The first important inhomogeneity among these studies is that among a total of 28 cohort studies, eight had been conducted on patients with BSIs caused only by ESBL-producing Enterobacteriaceae [9,18,43,46-50], whereas the remaining 20 were conducted on patients with BSIs caused by both ESBL- and non-ESBL-producing Enterobacteriaceae [13,15,19,20,26,28-31,33,34,44,45,51-57]; of course, the ESBL production by bacterial isolates could be included as a variable in the statistical analysis for prognostic factors only in the latter studies.

In addition, the species of Enterobacteriaceae included are different between the studies: ten studies had been conducted on patients with Ec BSIs [9,13,15,29,33,47,51,53,56,57], six on patients with Kp BSIs [18,19,32,36,37,39], ten on patients with different species of Enterobacteriaceae $[20,26,30,31,43,45,48,50,51,55]$, one on patients with E. cloacae BSIs [34] and one with Pm BSIs [28]. Only eight of the 28 studies were prospective $[9,13,15,26,30,49,52,53]$ and seven were multicenter studies $[9,13,30,31,43,45,49]$, whereas the others were retrospective and conducted in single centers. Lastly, five studies included nosocomial BSIs only $[9,20,26,49,52]$ and six included communityacquired BSIs only $[13,29,30,33,55,56]$, whereas the other 12 studies included cases with any type of BSI acquisition. Finally, two studies were conducted specifically in patients suffering from hematological malignancies $[31,57]$.

Several studies have investigated the impact of ESBL production by Enterobacteriaceae isolates on outcome of infections caused by these bacteria, and discordant results have been reported. Schwaber et al. have conducted a systematic review and meta-analysis of studies published through to April 2006 in order to examine the impact of ESBL production on mortality and delay in effective therapy in patients with Enterobacteriaceae BSIs, demonstrating a significantly increased rate of mortality (pooled relative risk: 1.85; 95\% CI: 1.39-2.47) in the ESBL group [7]. Among the 20 studies conducted on patients with BSIs caused by both ESBL- and non-ESBLproducing Enterobacteriaceae included in the 


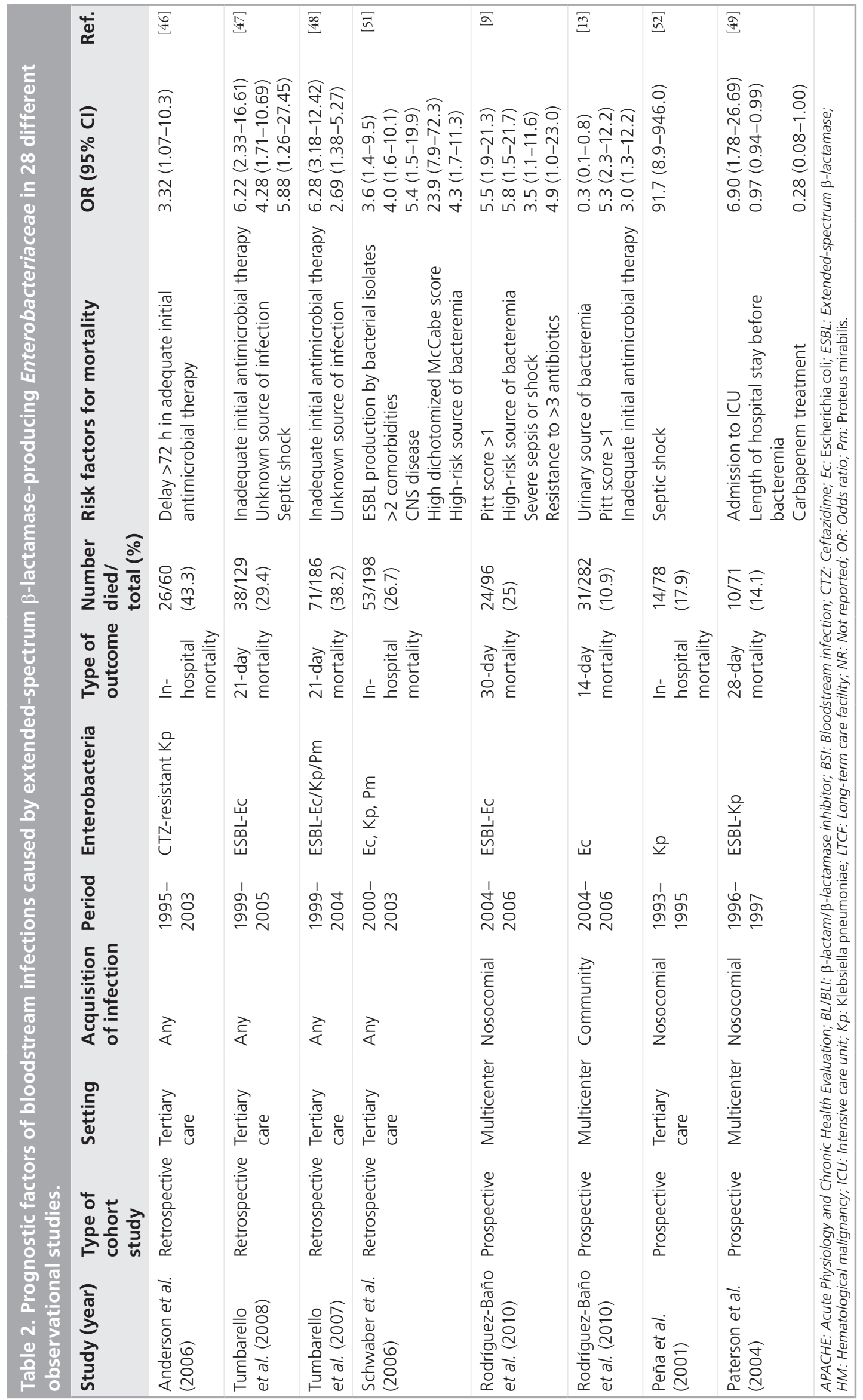




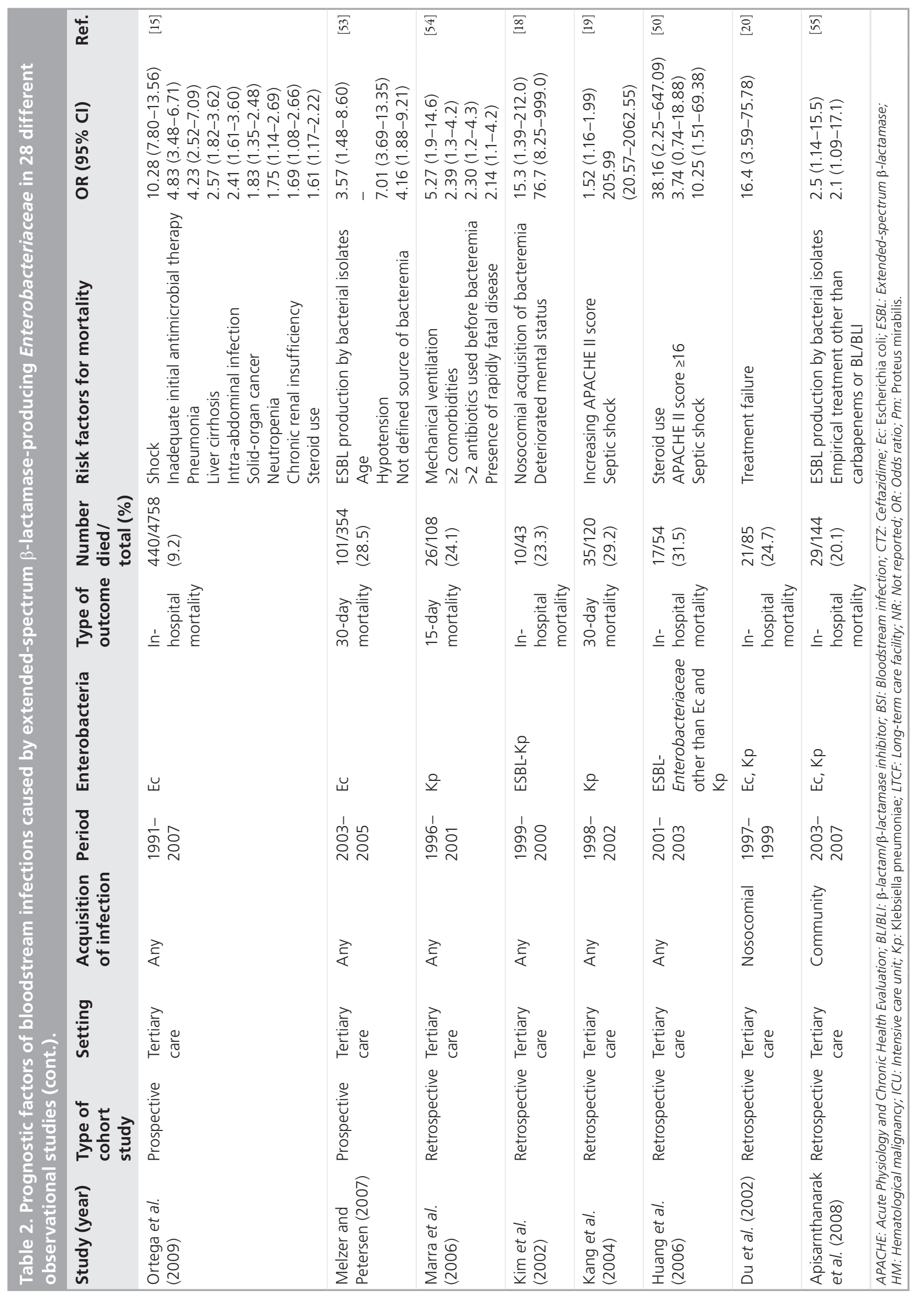




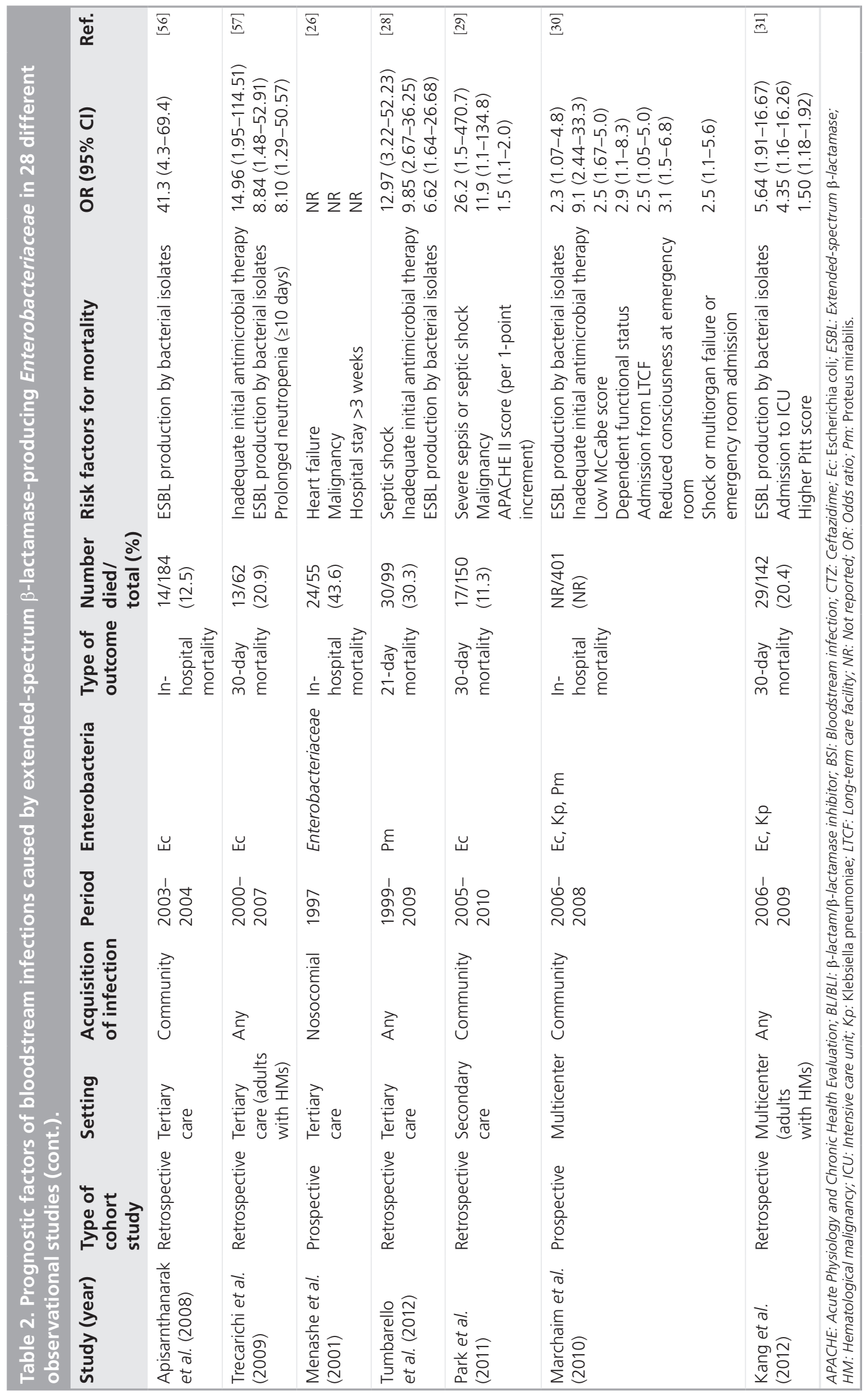




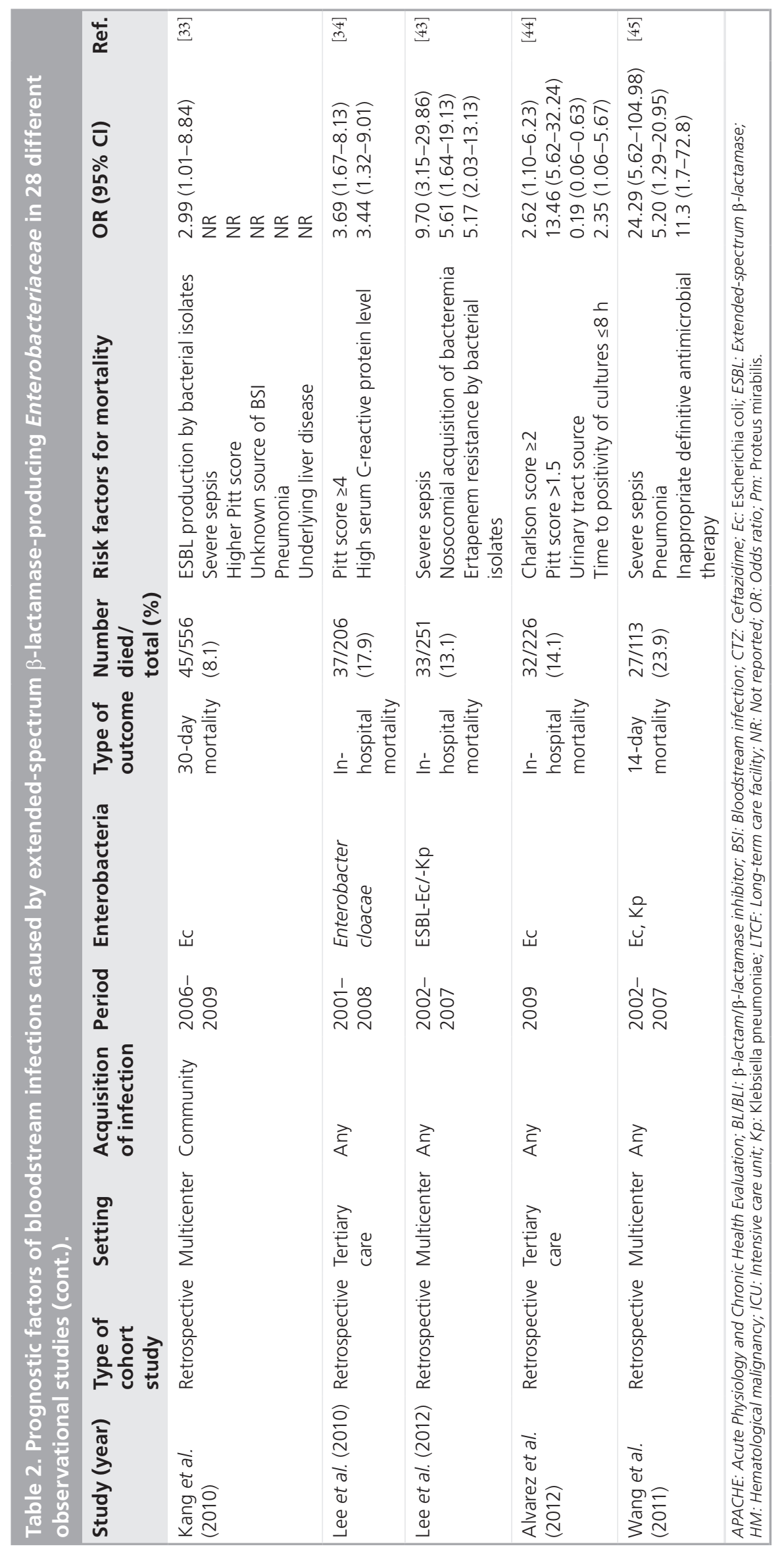


present review [13,15,19,20,26,28-31,33,34,44,45,51-57], ESBL production by bacterial isolates was independently associated with mortality in logistic regression analysis in only nine studies $[28,30,31,33,51,53,55-57]$, with ORs ranging from 2.3 to 41.3 . These data might appear in contradiction with the findings reported in the metaanalysis of Schwaber et al. [7]; however, these authors also found that only approximately half of the studies showed a significant association between ESBL production by the isolates and mortality, and attributed this finding to small sample sizes and, subsequently, to a lack of sufficient statistical power in the majority of the studies analyzed [7]. In addition, they calculated that to detect a significant association between ESBL production and mortality in a single study would require a sample size of 342 patients; among the studies published after the metaanalysis, four had a sample size greater than 342 cases: the study of Ortega and colleagues [15] included a cohort of $4758(26 \%)$ patients, that of Marchaim et al. [30] 401 patients, that of Kang et al. had 556 patients [33] and that of Melzer and Petersen had 354 patients [53]. It is of note that in all these studies except that of Ortega et al. [15], ESBL production by the isolates was independently associated with mortality.

One of the most frequently reported risk factors for mortality in patients with Enterobacteriaceae BSIs is the delay in effective antimicrobial therapy, which was found in the meta-analysis of Schwaber et al. [7] to be increased by approximately fivefold (pooled relative risk: 5.36; 95\% CI: 2.73-10.53) in the ESBL group.

Among the 28 studies on prognostic factors selected for this review, the inadequacy of initial antimicrobial therapy has been assessed (and included in logistic models) in 23 of them $[9,13,15,18,20,28-31,33,34,43-50,52,54,55,57]$; among these, this factor was independently associated with mortality in nine studies, with ORs ranging from 2.1 to 14.9 [13,15,28,30,46-48,55,57]. Conversely, use of a carbapenem (i.e., an appropriate antibiotic treatment) during the 5-day period after onset of bacteremia due to an ESBL-producing organism was independently associated with lower mortality in the study of Paterson et al. [49]; in the study of Apisarnthanarak et al. [56], the logistic regression model was adjusted for the inadequate initial antibiotic therapy, thus excluding this variable from the analysis; and in the studies of Du et al. [20] and Melzer and Petersen [53], the mortality rate was significantly higher in the group of patients that was inadequately treated compared with the adequately treated group; however, when the variable of inadequate initial antibiotic therapy was included in the logistic model for prognostic factors, it was not significant in the study of Du et al., whereas it was apparently not included in the logistic model in the study of Melzer et al. In the other 12 studies, inappropriate initial antimicrobial treatment was included in the analysis and no association with mortality was found $[9,18,29,31,33,34,43-45,50,52,54]$; however, the relatively small sample sizes of most of these studies could explain in large part these results. Obviously, ESBL production and inadequate empirical therapy are greatly correlated; for this reason, it is very likely that by including both of these variables in multivariable models, one can exclude the other from the model. In Figure 1 , all of the studies including these variables in multivariable models have been reported in a tree diagram, according to inclusion of one or both of these variables, and the results of the statistical analyses for these variables are shown.

With regard to the adequacy of antibiotic treatment, carbapenems are generally considered to be the drug of choice for the treatment of BSIs caused by ESBL-producing Enterobacteriaceae. Interestingly, Rodríguez-Baño and colleagues conducted a post hoc analysis of patients with ESBL-producing Ec BSIs from six published prospective cohorts, demonstrating no significant differences in terms of mortality and length of hospital stay between patients treated, either empirically or definitively, with an active $\beta$-lactam/ $\beta$-lactam inhibitor (i.e., amoxicillin-clavulanic acid and piperacillin-tazobactam) and carbapenems, thus suggesting that $\beta$-lactam/ $\beta$-lactam inhibitors would be useful alternatives to carbapenems for these patients, in particular for definitive therapy [58].

Two studies evaluated risk factors for delay in appropriate initial antimicrobial therapy in patients with BSIs caused by Enterobacteriaceae [47,51]. Schwaber et al. found that ESBL production was the unique significant predictor of delay in appropriate therapy (OR: 25.1) in their cohort of patients with Enterobacteriaceae BSIs [51], and Tumbarello et al. determined that unknown BSI source (OR: 4.86), isolate coresistance to more than three antimicrobials (OR: 3.73), hospitalization during the 12 months preceding BSI onset (OR: 3.33) and antimicrobial therapy during the 3 months preceding BSI onset (OR: 2.65) were independent risk factors for inadequate initial antimicrobial therapy in a cohort of patients with BSIs caused by ESBL-producing Ec [47]. 


\section{Review Trecarichi, Cauda \& Tumbarello}

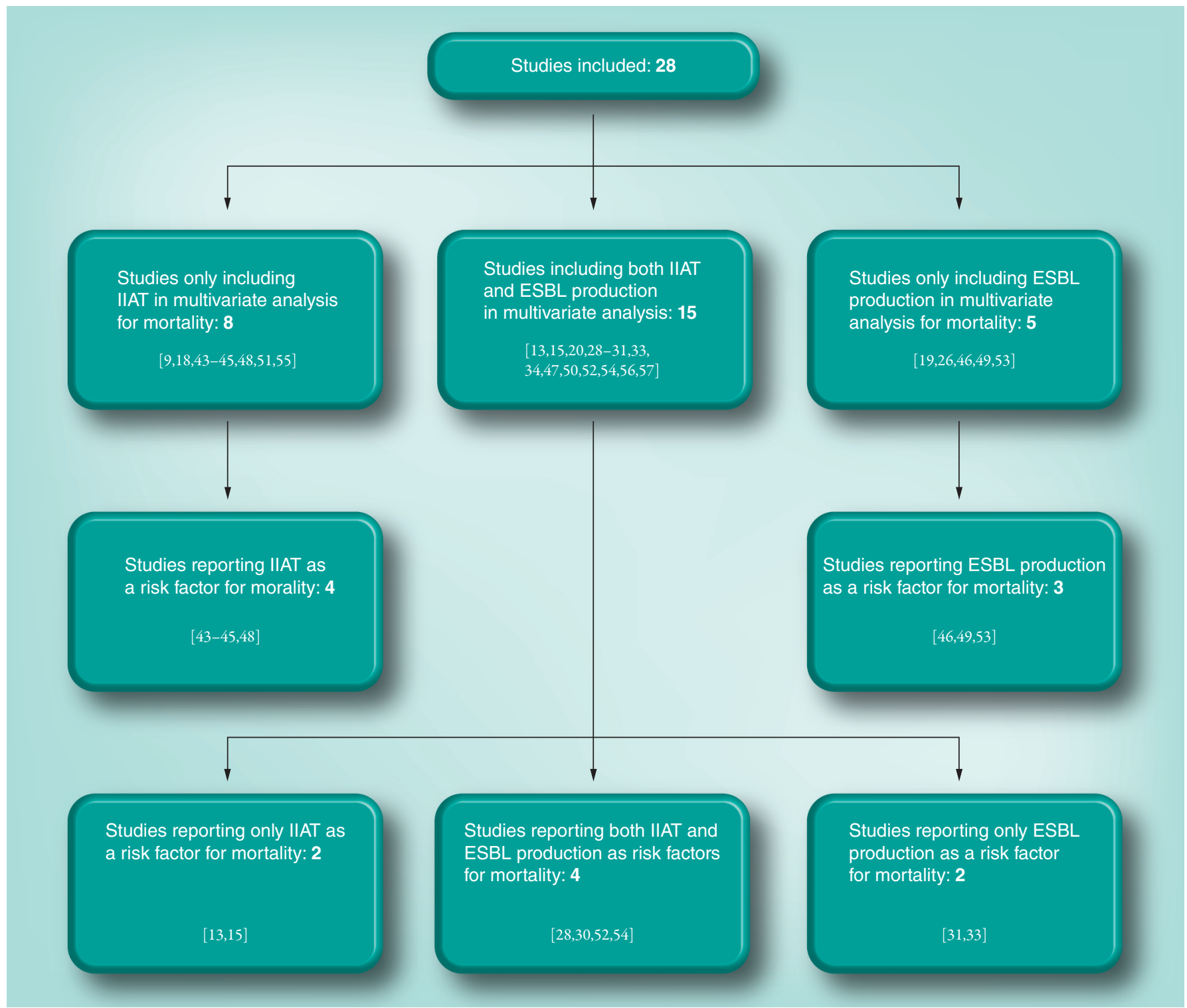

Figure 1. Tree diagram categorizing all of the studies on risk factors for mortality in patients with extended-spectrum $\beta$-lactamase-producing Enterobacteriaceae, according to inclusion in multivariable models of the variables 'inadequate initial antimicrobial therapy' and/or 'extended-spectrum $\beta$-lactamase production by bacterial isolates', as well as according to results of statistical analyses for these variables.

ESBL: Extended-spectrum $\beta$-lactamase; IIAT: Inadequate initial antimicrobial therapy.

Factors related to the clinical severity of the BSIs, which may sometimes be intermediates (reducing the effect of ESBL production or other factors on mortality) [42], were among the factors most frequently associated with mortality: septic shock, severe sepsis or hypotension have been found to be independently associated with mortality in 12 studies [9,19,28-30,33,43,45,47,50,52,53]; admission to an ICU or mechanical ventilation were independently associated with mortality in three studies [31,49,54]; and deteriorating mental status was independently associated with mortality in two studies $[18,30]$. In addition, the overall severity of the patient's illness, expressed as the Acute Physiology and Chronic Health Evaluation (APACHE) II score or the Pitt bacteremia score, was reported and associated with mortality in nine studies $[9,13,19,29,31,33,34,44,50]$, with a reported threshold of $\geq 16$ and $>1$ points for APACHE II and Pitt scores, respectively, identifying patients with a high risk for mortality $[9,13,50]$.

A number of underlying diseases or factors related to the patient's clinical status have been reported as being independently associated with mortality, such as age, CNS disease, pneumonia, liver cirrhosis, malignancy, neutropenia, chronic renal insufficiency and heart 
failure $[15,26,28,29,33,43,45,53,57]$. The presence of more than two comorbidities or a rapidly fatal disease as classified by McCabe and Jackson criteria or a Charlson Comorbidity Index $>2$ were found to be associated with mortality in four studies [30,44,51,54], as well as corticosteroid therapy in two other studies $[15,50]$.

In eight studies, the role of the source of BSI on mortality has been highlighted; in particular, unknown source of BSI or abdominal or respiratory tract infection (in some studies defined as 'high-risk source of BSI') have been related to adverse outcome $[9,33,47,48,51,53]$; conversely, a urinary source of BSI has been associated with a high probability of survival $[13,44]$.

Finally, Alvarez et al. analyzed the effect of time to positivity of blood cultures on outcomes in patients with Ec BSIs and demonstrated that a lower time to positivity $(<8 \mathrm{~h})$ is an independent risk factor for septic shock and poor outcome [44].

\section{Future perspective}

Medical doctors and researchers should be aware of the increasing prevalence of ESBL-producing organisms and, in particular, of the changing epidemiology with the recent emergence of community-onset severe infections caused by these bacteria, which make these infections an important public health problem.

Any delay in the initiation of adequate antibiotic therapy is potentially lethal for patients with BSIs caused by ESBL-producing Enterobacteriaceae. The awareness of changes in bacterial resistance patterns, the considered knowledge of risk factors for ESBL infection and of factors facilitating adverse outcome, and attention to the local epidemiology can improve the efficacy of empirical treatment protocols.

Financial \& competing interests disclosure
The authors have no relevant affiliations or financial
involvement with any organization or entity with a
financial interest in or financial conflict with the sub-
ject matter or materials discussed in the manuscript.
This includes employment, consultancies, honoraria,
stock ownership or options, expert testimony, grants or
patents received or pending, or royalties.
No writing assistance was utilized in the production
of this manuscript.

\section{Executive summary}

\section{Background}

- Extended-spectrum $\beta$-lactamases (ESBLs) are plasmid-mediated $\beta$-lactamases that confer resistance to oxyimino-cephalosporins and monobactams and have been increasingly described worldwide, especially among Enterobacteriaceae isolates.

- Recently, ESBL-producing Enterobacteriaceae have been found to cause infections with increasing frequency in the community setting.

Risk factors for bloodstream infections caused by ESBL-producing Enterobacteriaceae

- Recent antibiotic therapy, particularly with $\beta$-lactams, has been reported as one of the most important risk factors for ESBL-producing Enterobacteriaceae bloodstream infections (BSIs).

- Presence of comorbidities and previous invasive procedures and/or presence of devices have been recognized as predicting BSIs caused by ESBL-producing Enterobacteriaceae, for both community- and nosocomial-acquired infections.

- Admission from long-term care facilities has been found to be strongly associated with non-nosocomial ESBL-producing Enterobacteriaceae BSIs.

Predictors of mortality in patients with BSIs caused by ESBL-producing Enterobacteriaceae

- The delay in effective antimicrobial therapy is one of the most frequently reported risk factors for mortality in patients with ESBL-producing Enterobacteriaceae BSIs, together with factors related to severity of comorbidities and of a patient's clinical status.

\section{References}

Papers of special note have been highlighted as:

- of interest

- of considerable interest

1. Rodríguez-Baño J, Pascual A. Clinical significance of extended-spectrum betalactamases. Expert Rev. Anti Infect. Ther. 6(5), 671-683 (2008).

- Comprehensive review of the epidemiology, clinical aspects and treatment options of infections caused by extended-spectrum$\beta$-lactamase (ESBL)-producing Enterobacteriaceae.
2. Paterson DL, Bonomo RA. Extendedspectrum beta-lactamases: a clinical update. Clin. Microbiol. Rev. 18(4), 657-686 (2005).

-. Comprehensive review of the epidemiology, clinical aspects and treatment options of infections caused by ESBL-producing Enterobacteriaceae.

3. Pitout JD. Infections with extended-spectrum beta-lactamase-producing Enterobacteriaceae: changing epidemiology and drug treatment choices. Drugs 70 (3), 313-333 (2010).

-. Comprehensive review of the epidemiology and treatment options of infections caused by ESBL-producing Enterobacteriaceae.

4. Giske CG, Monnet DL, Cars O, Carmeli Y; ReAct-Action on Antibiotic Resistance. Clinical and economic impact of common multidrugresistant Gram-negative bacilli. Antimicrob. Agents Chemother. 52(3), 813-821 (2008).

- Exhaustive review on the clinical and economic impact of infections caused by common multidrug-resistant Gram-negative bacilli, including ESBL producers.

5. Tumbarello M, Spanu T, Di Bidino R et al. Costs of bloodstream infections caused by 
Escherichia coli and influence of extendedspectrum-beta-lactamase production and inadequate initial antibiotic therapy. Antimicrob. Agents Chemother. 54(10), 4085-4091 (2010).

- Evidence of the important impact of ESBL production and inadequate initial antibiotic therapy on hospital costs of Escherichia coli bloodstream infections.

6. Schwaber MJ, Navon-Venezia S, Schwartz D, Carmeli Y. High levels of antimicrobial coresistance among extended-spectrum-betalactamase-producing Enterobacteriaceae. Antimicrob. Agents Chemother. 49(5), 2137-2139 (2005).

7. Schwaber MJ, Carmeli Y. Mortality and delay in effective therapy associated with extendedspectrum beta-lactamase production in Enterobacteriaceae bacteraemia: a systematic review and meta-analysis. J. Antimicrob. Chemother. 60 (5), 913-920 (2007).

8. Tumbarello M, Spanu T, Sanguinetti M et al. Bloodstream infections caused by extendedspectrum-beta-lactamase-producing Klebsiella pneumoniae: risk factors, molecular epidemiology, and clinical outcome. Antimicrob. Agents Chemother. 50(2), 498-504 (2006).

9. Rodríguez-Baño J, Picón E, Gijón P et al. Risk factors and prognosis of nosocomial bloodstream infections caused by extendedspectrum-beta-lactamase-producing Escherichia coli. J. Clin. Microbiol. 48(5), 1726-1731 (2010).

10. Wu UI, Yang CS, Chen WC, Chen YC, Chang SC. Risk factors for bloodstream infections due to extended-spectrum beta-lactamase-producing Escherichia coli. J. Microbiol. Immunol. Infect. 43(4), 310-316 (2010).

11. Superti SV, Augusti G, Zavascki AP. Risk factors for and mortality of extendedspectrum-beta-lactamase-producing Klebsiella pneumoniae and Escherichia coli nosocomial bloodstream infections. Rev. Inst. Med. Trop. Sao Paulo 51(4), 211-216 (2009).

12. Skippen I, Shemko M, Turton J, Kaufmann ME, Palmer C, Shetty N. Epidemiology of infections caused by extended-spectrum beta-lactamase-producing Escherichia coli and Klebsiella spp.: a nested case-control study from a tertiary hospital in London. J. Hosp. Infect. 64(2), 115-123 (2006).

13. Rodríguez-Baño J, Picón E, Gijón P et al. Community-onset bacteremia due to extended-spectrum beta-lactamase-producing Escherichia coli: risk factors and prognosis. Clin. Infect. Dis. 50 (1), 40-48 (2010).

14. Qureshi ZA, Paterson DL, Pakstis DL et al. Risk factors and outcome of extendedspectrum $\beta$-lactamase-producing
Enterobacter cloacae bloodstream infections. Int. J. Antimicrob. Agents 37(1), 26-32 (2011).

15. Ortega M, Marco F, Soriano A et al. Analysis of 4758 Escherichia coli bacteraemia episodes: predictive factors for isolation of an antibioticresistant strain and their impact on the outcome. J. Antimicrob. Chemother. 63(3), 568-574 (2009).

16. Mosqueda-Gómez JL, Montaño-Loza A, Rolón AL et al. Molecular epidemiology and risk factors of bloodstream infections caused by extended-spectrum beta-lactamase-producing Klebsiella pneumoniae: a case-control study. Int. J. Infect. Dis. 12 (6), 653-659 (2008).

17. Kim YK, Pai H, Lee HJ et al. Bloodstream infections by extended-spectrum betalactamase-producing Escherichia coli and Klebsiella pneumoniae in children: epidemiology and clinical outcome. Antimicrob. Agents Chemother. 46(5), 1481-1491 (2002).

18. Kim BN, Woo JH, Kim MN, Ryu J, Kim YS. Clinical implications of extended-spectrum beta-lactamase-producing Klebsiella pneumoniae bacteraemia. J. Hosp. Infect. 52(2), 99-106 (2002).

19. Kang CI, Kim SH, Kim DM et al. Risk factors for and clinical outcomes of bloodstream infections caused by extendedspectrum beta-lactamase-producing Klebsiella pneumoniae. Infect. Control Hosp. Epidemiol. 25(10), 860-867 (2004).

20. Du B, Long $\mathrm{Y}$, Liu $\mathrm{H}$ et al. Extendedspectrum beta-lactamase-producing Escherichia coli and Klebsiella pneumoniae bloodstream infection: risk factors and clinical outcome. Intensive Care Med. 28(12), 1718-1723 (2002).

21. Ben-Ami R, Schwaber MJ, Navon-Venezia S et al. Influx of extended-spectrum betalactamase-producing Enterobacteriaceae into the hospital. Clin. Infect. Dis. 42(7), 925-934 (2006).

22. Ariffin $\mathrm{H}, \mathrm{Navaratnam} \mathrm{P}$, Mohamed $\mathrm{M}$ et al. Ceftazidime-resistant Klebsiella pneumoniae bloodstream infection in children with febrile neutropenia. Int. J. Infect. Dis. 4(1), 21-25 (2000).

23. Zaoutis TE, Goyal M, Chu JH et al. Risk factors for and outcomes of bloodstream infection caused by extended-spectrum beta-lactamase-producing Escherichia coli and Klebsiella species in children. Pediatrics 115(4), 942-949 (2005).

24. Paterson DL, Ko WC, Von Gottberg A et al. International prospective study of Klebsiella pneumoniae bacteremia: implications of extended-spectrum beta-lactamase production in nosocomial infections. Ann. Intern. Med. 140(1), 26-32 (2004).
25. Behar PR, Teixeira PJ, Fachel JM, Kalil AC. The effect of control group selection in the analysis of risk factors for extended spectrum beta-lactamase-producing Klebsiella pneumoniae infections. A prospective controlled study. J. Hosp. Infect. 68(2), 123-129 (2008).

26. Menashe G, Borer A, Yagupsky P et al. Clinical significance and impact on mortality of extended-spectrum beta lactamaseproducing Enterobacteriaceae isolates in nosocomial bacteremia. Scand. J. Infect. Dis. 33(3), 188-193 (2001).

27. Ho PL, Chan WM, Tsang KW, Wong SS, Young K. Bacteremia caused by Escherichia coli producing extended-spectrum betalactamase: a case-control study of risk factors and outcomes. Scand. J. Infect. Dis. 34(8), 567-573 (2001).

28. Tumbarello M, Trecarichi EM, Fiori B et al. Multidrug-resistant Proteus mirabilis bloodstream infections: risk factors and outcomes. Antimicrob. Agents Chemother. 56(6), 3224-3231 (2012).

29. Park SH, Choi SM, Lee DG et al. Emergence of extended-spectrum $\beta$-lactamase-producing Escherichia coli as a cause of community-onset bacteremia in South Korea: risk factors and clinical outcomes. Microb. Drug Resist. 17(4), 537-544 (2011).

30. Marchaim D, Gottesman T, Schwartz O et al. National multicenter study of predictors and outcomes of bacteremia upon hospital admission caused by Enterobacteriaceae producing extended-spectrum betalactamases. Antimicrob. Agents Chemother. 54(12), 5099-5104 (2010).

31. Kang CI, Chung DR, Ko KS, Peck KR, Song JH; Korean Network for Study of Infectious Diseases. Risk factors for infection and treatment outcome of extended-spectrum $\beta$-lactamase-producing Escherichia coli and Klebsiella pneumoniae bacteremia in patients with hematologic malignancy. Ann. Hematol. 91(1), 115-121 (2012).

32. Cornejo-Juárez P, Pérez-Jiménez C, Silva-Sánchez J et al. Molecular analysis and risk factors for Escherichia coli producing extended-spectrum $\beta$-lactamase bloodstream infection in hematological malignancies. PLoS One 7(4), e35780 (2012).

33. Kang CI, Song JH, Chung DR et al. Risk factors and treatment outcomes of community-onset bacteraemia caused by extended-spectrum beta-lactamase-producing Escherichia coli. Int. J. Antimicrob. Agents 36(3), 284-287 (2010).

34. Lee CC, Lee NY, Yan JJ et al. Bacteremia due to extended-spectrum-beta-lactamaseproducing Enterobacter cloacae: role of carbapenem therapy. Antimicrob. Agents Chemother. 54(9), 3551-3556 (2010). 
35. Freeman JT, McBride SJ, Nisbet MS et al. Bloodstream infection with extendedspectrum beta-lactamase-producing Enterobacteriaceae at a tertiary care hospital in New Zealand: risk factors and outcomes. Int. J. Infect. Dis. 16(5), e371-e374 (2012).

36. Quirante OF, Cerrato SG, Pardos SL. Risk factors for bloodstream infections caused by extended-spectrum $\beta$-lactamase-producing Escherichia coli and Klebsiella pneumoniae. Braz. J. Infect. Dis. 15(4), 370-376 (2011).

37. Tuon FF, Kruger M, Terreri M, PenteadoFilho SR, Gortz L. Klebsiella ESBL bacteremia-mortality and risk factors. Braz. J. Infect. Dis. 15(6), 594-598 (2011).

38. Wu UI, Wang JL, Chen WC, Chang SC, Chen YC. Risk factors and outcomes of Escherichia coli bacteremia caused by strains that produce CTX-M or non-CTX-M extended-spectrum-beta-lactamases. Eur. J. Clin. Microbiol. Infect. Dis. 30(1), 33-39 (2011).

39. Harris AD, Karchmer TB, Carmeli Y, Samore $\mathrm{MH}$. Methodological principles of casecontrol studies that analyzed risk factors for antibiotic resistance: a systematic review. Clin. Infect. Dis. 32(7), 1055-1061 (2001).

- Interesting systematic review of casecontrol studies analyzing risk factors for the development of antibiotic-resistant organisms and addressing methodological principles of control group selection and adjustment for confounding factors.

40. Tumbarello M, Trecarichi EM, Bassetti M et al. Identifying patients harboring extended-spectrum-beta-lactamase-producing Enterobacteriaceae on hospital admission: derivation and validation of a scoring system. Antimicrob. Agents Chemother. 55(7), 3485-3490 (2011).

41. Nicolas-Chanoine MH, Jarlier V. Extendedspectrum beta-lactamases in long-term-care facilities. Clin. Microbiol. Infect. 14(Suppl. 1), 111-116 (2008).

42. Rottier WC, Ammerlaan HS, Bonten MJ. Effects of confounders and intermediates on the association of bacteraemia caused by extended-spectrum $\beta$-lactamase-producing Enterobacteriaceae and patient outcome: a meta-analysis. J. Antimicrob. Chemother. 67(6), 1311-1320 (2012).

43. Lee NY, Lee CC, Huang WH, Tsui KC, Hsueh PR, Ko WC. Carbapenem therapy for bacteremia due to extended-spectrum- $\beta$ lactamase-producing Escherichia coli or
Klebsiella pneumoniae: implications of ertapenem susceptibility. Antimicrob. Agents Chemother. 56(6), 2888-2893 (2012).

44. Alvarez R, Viñas-Castillo L, Lepe-Jiménez JA, García-Cabrera E, Cisneros-Herreros JM. Time to positivity of blood culture association with clinical presentation, prognosis and ESBL-production in Escherichia coli bacteremia. Eur. J. Clin. Microbiol. Infect. Dis. 31(9), 2191-2195 (2012).

45. Wang SS, Lee NY, Hsueh PR et al. Clinical manifestations and prognostic factors in cancer patients with bacteremia due to extended-spectrum $\beta$-lactamase-producing Escherichia coli or Klebsiella pneumoniae. J. Microbiol. Immunol. Infect. 44(4), 282-288 (2011).

46. Anderson DJ, Engemann JJ, Harrell LJ, Carmeli Y, Reller LB, Kaye KS. Predictors of mortality in patients with bloodstream infection due to ceftazidime-resistant Klebsiella pneumoniae. Antimicrob. Agents Chemother. 50(5), 1715-1720 (2006).

47. Tumbarello M, Sali M, Trecarichi EM et al. Bloodstream infections caused by extendedspectrum-beta-lactamase-producing Escherichia coli: risk factors for inadequate initial antimicrobial therapy. Antimicrob. Agents Chemother. 52(9), 3244-3252 (2008).

48. Tumbarello M, Sanguinetti M, Montuori E et al. Predictors of mortality in patients with bloodstream infections caused by extendedspectrum-beta-lactamase-producing Enterobacteriaceae: importance of inadequate initial antimicrobial treatment. Antimicrob. Agents Chemother. 51(6), 1987-1994 (2007).

49. Paterson DL, Ko WC, Von Gottberg A et al. Antibiotic therapy for Klebsiella pneumoniae bacteremia: implications of production of extended-spectrum beta-lactamases. Clin. Infect. Dis. 39(1), 31-37 (2004).

50. Huang SS, Lee MH, Leu HS. Bacteremia due to extended-spectrum beta-lactamaseproducing Enterobacteriaceae other than Escherichia coli and Klebsiella. J. Microbiol. Immunol. Infect. 39(6), 496-502 (2006).

51. Schwaber MJ, Navon-Venezia S, Kaye KS, Ben-Ami R, Schwartz D, Carmeli Y. Clinical and economic impact of bacteremia with extended-spectrum-beta-lactamase-producing Enterobacteriaceae. Antimicrob. Agents Chemother. 50(4), 1257-1262 (2006).

52. Peña C, Pujol M, Ardanuy C et al. An outbreak of hospital-acquired Klebsiella pneumoniae bacteraemia, including strains producing extended-spectrum beta-lactamase. J. Hosp. Infect. 47(1), 53-59 (2001).

53. Melzer M, Petersen I. Mortality following bacteraemic infection caused by extended spectrum beta-lactamase (ESBL) producing E. coli compared to non-ESBL producing E. coli. J. Infect. 55(3), 254-259 (2007).

54. Marra AR, Wey SB, Castelo A et al. Nosocomial bloodstream infections caused by Klebsiella pneumoniae: impact of extendedspectrum beta-lactamase (ESBL) production on clinical outcome in a hospital with high ESBL prevalence. BMC Infect. Dis. 6, 24 (2006).

55. Apisarnthanarak A, Kiratisin P, Mundy LM. Predictors of mortality from communityonset bloodstream infections due to extended-spectrum beta-lactamase-producing Escherichia coli and Klebsiella pneumoniae. Infect. Control Hosp. Epidemiol. 29(7), 671-674 (2008).

56. Apisarnthanarak A, Kiratisin P, Mundy LM. Clinical and molecular epidemiology of healthcare-associated infections due to extended-spectrum beta-lactamase (ESBL)producing strains of Escherichia coli and Klebsiella pneumoniae that harbor multiple ESBL genes. Infect. Control Hosp. Epidemiol. 29(11), 1026-1034 (2008).

57. Trecarichi EM, Tumbarello M, Spanu T et al. Incidence and clinical impact of extendedspectrum-beta-lactamase (ESBL) production and fluoroquinolone resistance in bloodstream infections caused by Escherichia coli in patients with hematological malignancies. J. Infect. 58(4), 299-307 (2009).

58. Rodríguez-Baño J, Navarro MD, Retamar P, Picón E, Pascual Á; Extended-Spectrum Beta-Lactamases-Red Española de Investigación en Patología Infecciosa/Grupo de Estudio de Infección Hospitalaria Group. $\beta$-lactam/ $\beta$-lactam inhibitor combinations for the treatment of bacteremia due to extended-spectrum $\beta$-lactamase-producing Escherichia coli: a post hoc analysis of prospective cohorts. Clin. Infect. Dis. 54(2), 167-174 (2012).

\section{Website}

101. European Antimicrobial Resistance Surveillance System (EARSS), Annual Report 2010. www.ecdc.europa.eu/en/publications/ Publications/1111_SUR_AMR_data.pdf 